\title{
Biopolitica dell'igiene nel primo dopoguerra. Genere e governo dei corpi nella costruzione dell'assistente sanitaria visitatrice ${ }^{1}$
}

\author{
Olivia Fiorilli
}

Nel maggio 1923, di ritorno dalla conferenza internazionale organizzata a Parigi dallo European Council of Nursing Education - organizzazione nata sotto l'egida dell'American Red Cross con l'intento di coordinare i programmi di educazione delle infermiere in Europa - Irene di Targiani Giunti, ispettrice generale delle infermiere della Croce rossa italiana e presidente dell'Associazione nazionale tra infermiere, scriveva su "Difesa sociale": "la prevalenza nelle discussioni venne beninteso data al problema delle infermiere visitatrici; su queste può dirsi è fondata la grande riforma sociale e igienica futura"². L'affermazione di Targiani Giunti non appare iperbolica se si esaminano i discorsi sul governo della salute della popolazione che saturavano il dibattito sulle misure da prendere dopo la Grande guerra tanto in Italia quanto a livello internazionale. Di assistenti sanitarie visitatrici, visiting nurses, public health nurses, infirmières visiteuses si faceva, infatti, un gran parlare nei convegni internazionali ${ }^{3}$, mentre la formazione di queste figure, iniziata in diversi stati pro-

${ }^{1}$ Parte delle ricerche che sono alla base di questo articolo, in particolare quelle svolte presso gli Hoover Institution Archives di Stanford, CA-Usa, sono state possibili grazie all'Agnodike Research Travel Fellowship accordatami dalla Commission on Women and Gender Studies in Science, Technology and Medicine della Division of History of Science and Technology of the International Union of History and Philosophy of Science. Il resto delle ricerche sono state possibili grazie alla borsa dottorale conferitami dal Dottorato internazionale in Studi di genere dell'Università di Roma "Sapienza".

${ }^{2}$ Irene di Targiani Giunti, Relazione sul congresso internazionale di Parigi per l'educazione delle infermiere, "Difesa sociale", maggio 1923, n. 5, p. 75. Sul convegno dello European Council of Nursing Education tenutosi a Parigi nel 1923 si veda Jaime Patricia Lapeyre, "The Idea of Better Nursing": the American Battle for Contro Lover Standards of Nursing Education in Europe, 1915-1925, PhD dissertation, Graduate Department of Nursing Science, University of Toronto, 2013, pp. $5 \mathrm{sg}$.

${ }^{3}$ Un esempio tra tutti: la conferenza tenutasi a Cannes nel 1919, per volere dell'American Red Cross dalla quale nacque la League of Red Cross Societies, dedicò alla questione del public health nursing un'ampia sezione: "Proceedings of the medical conference held at the invita- 
prio in quegli anni ${ }^{4}$, era un punto qualificante dei programmi avviati in molti paesi dagli organismi internazionali e dalle organizzazioni filantropiche nordamericane (League of Nations Health Organization, League of Red Cross Societies, Rockefeller Foundation, American Red Cross) $)^{5}$ che nel primo dopoguerra facevano il loro ingresso nell'arena del global health ${ }^{6}$. Le public health nurses - o assistenti sanitarie visitatrici - erano infatti figure incaricate primariamente ${ }^{7}$ di

tion of the Committee of Red Cross Societies, april 1 to 11 1919", Cannes, League of Red Cross Societies, 1919. Si veda anche Ettore Levi, La difesa dei valori umani. Organizzazione nazionale ed internazionale di igiene e assistenza sociale, Roma, Istituto italiano d'igiene, previdenza e assistenza sociale, 1924.

${ }^{4}$ Josep Bernabeu Mestre, Encarna Gascon Pérez, El papel de la enfermería en el desarrollo de la salud pública española (1923-1925): la visitadora sanitaria, "Dynamis", (15) 1995, pp. 151-176; Yvonne Knibiehler, La "lutte antituberculeuse" instrument de la médicalisation des classes populaires (1870-1930), "Annales de Bretagne et des pays de l'Ouest", 1979, n. 2, pp. 321-336 DOI: 10.3406/abpo.1979.2986; Stephane Henry, Les infirmières-visiteuses pendant l'entre-deux-guerres en Haute-Normandie: entre professionnalisme officiel et bénévolat officieux, "Genre \& histoire. La revue de l'association Mnémosyne", 2009, n. 5, online all'indirizzo web https://genrehistoire.revues.org/836, ultimo accesso agosto 2015; Kristina Popova, $\mathrm{Be}$ tween Public Health and Social Work: Visiting Nurses in the Struggle against Poverty and Infant Mortality in Bulgaria 1923-1934, "Social Work and Society. International Online Journal", 2011, n. 2; Ead., From Visiting Ladies Towards Municipality Female Social Advisors: Women in Home Visiting Practice in Bulgaria (1915-1939), in Vesna Leskosek (a cura di), Teaching Gender in Social Work, Utrecht, Athena, 2009, pp. 127-145.

${ }^{5}$ Elisabeth Vickers, Frances Elizabeth Crowell and the Politics of Nursing in Czechoslovakia after the First World War, "Nursing History Review", (7) 1999, pp. 67-96; Dominique Dessertine, Les infirmières visiteuses de la lutte antituberculeuse en France (1900-1930), "Bulletin du centre Pierre Léon d'histoire économique et sociale", 1995, n. 2-3, pp. 55-63, anche all'indirizzo web http://bcpl.ish-lyon.cnrs.fr/1995/Femmes_soignantes/-1995_2_3_55.pdf; Pierre Yves Saunier, Ludovic Tournès, Philantropies croisées: a Joint Venture in Public Health at Lyon (1917-1940), "French History", 2009, n. 2, pp. 216-240, anche all'indirizzo web https://halshs. archives-ouvertes.fr/halshs-00394318/document; Ann Yrjälä, Public Health and Rockefeller Wealth: Alliance Strategies in the Early Formation of Finnish Public Health Nursing, Åbo Akademis Förlag, Åbo Akademy U.P., 2005, anche all'indirizzo web www.doria.fi/bitstream/handle/10024/4147/TMP.objres.11.pdf?sequence=2; Barbara Brush, The Rockefeller Agenda for American/Philippines Nursing Relations, in Anne Marie Rafferty, Jane Robinson, Ruth Elkan (a cura di), Nursing History and the Politics of Welfare, London-New York, Routledge, 1997, pp. 45-63; Sarah Elise Abrams, Seeking Jurisdiction: a Sociological Perspective on Rockefeller Foundation Activities in Nursing in the 1920's, in A.M. Rafferty, J. Robinson, R. Elkan (a cura di), Nursing History, cit., pp. 207-225.

${ }^{6}$ Paul Weindling (a cura di), International Health Organizations and Movements, 1918-39, Cambridge, Cambridge U.P., 1995; Susan Solomon, Lion Murard, Paul Zylberman (a cura di), Shifting Boundaries of Public Health Europe in the Twentieth Century, Rochester, NY-Usa, University of Rochester Press, 2008; Improving Public Health: the Interwar Period in Europe, "Dynamis", (28) 2008, num. mon. a cura di Paul Zylberman, Esteban Rodríguez-Ocaña; John Farley, To Cast out Disease: a History of the International Health Division of the Rockefeller Foundation (1913-51), Oxford-New York, Oxford U.P., 2004.

${ }^{7}$ In effetti il problema del bilanciamento tra le diverse funzioni delle public health nurses e conseguentemente la questione della formazione più adatta per queste figure professionali, era un nodo irrisolto e molto dibattuto al termine della prima guerra mondiale. Su questi argomenti si veda J. Lapeyre, "The Idea of Better Nursing”, cit. 
diffondere il "vangelo dell'igiene" tra la popolazione e insegnare le norme per condurre una vita "sana e produttiva". Punto focale della loro opera era la "penetrazione" nell'ambiente famigliare attraverso la visita domiciliare, che doveva coniugare la diffusione dell'igiene al dépistage ${ }^{8}$ delle malattie trasmissibili, l'indagine all'assistenza sociale e — all'occorrenza — sanitaria. Nella genealogia del public health nursing si possono annoverare tanto il district nursing anglosassone e il visiting nursing nordamericano, quanto più in generale le diverse forme della filantropia femminile nel mondo anglosassone ${ }^{9}$.

Alla fine della Prima guerra mondiale l'assistente sanitaria visitatrice fece la sua comparsa anche in Italia. Questa figura è stata sostanzialmente ignorata dalla storiografia italiana, probabilmente a causa del suo carattere "ibrido" che l'ha resa periferica rispetto agli ambiti di interesse tanto della storia del servizio sociale ${ }^{10}$ quanto di quella dell'assistenza infermieristica ${ }^{11}$ e della sanità.

${ }^{8}$ In ambito medico con questo termine si fa riferimento alla ricerca attiva, all'interno di una popolazione, di soggetti affetti da una malattia in fase precoce oppure di fattori di rischio per patologie specifiche.

${ }^{9}$ Sulle origini del public health nursing si veda Karen Buhler Wilkerson, False Down: the Rise and Decline of Public Health Nursing 1900-1930, in Ellen Condliffe Lageman (a cura di), Nursing History: New Perspectives New Possibilities, New York, Teacher College Press, 1983, pp. 89-106; Ead., No Place like Home: a History of Nursing and Home Care in the United States, Oxford, Oxford U.P., 2001; Robert Dingwell, Anne Marie Rafferty, Charles Webster, An Introduction to the Social History of Nursing, London, Routledge, 1988.

${ }^{10}$ Maddalena Tirabassi, La nascita del servizio sociale in Italia: modelli statunitensi, radici italiane (1920-1950), in Dora Marucco (a cura di), Istituzioni e politiche sociali a Torino negli ultimi cinquant'anni. La cultura del servizio sociale, Torino, Celid, 2004, pp. 17-42; Pierangela Benvenuti, Domenica A. Gristina, La donna e il servizio sociale, identità professionale e sociale dell'assistenza sociale, Milano, FrancoAngeli, 1998.

${ }^{11}$ Sulla storia dell'assistenza infermieristica si vedano Stefania Bartoloni, La asistencia a los enferos en Italia: religiosa y laicas en la práctica de la enfermería, in Carmen González Canalejo, Fernando Martínez López (a cura di), La transformciòn de la enfermerìa. Nuevas miradas para la historia, Granada, Editorial Comares, 2010; Stefania Bartoloni, Carmen González Canalejo, Percorsi di formazione per 'l'infermiera moderna': Italia e Spagna 1870-1920, "Medicina \& storia", 2011, n. 21-22, pp. 39-61; Stefania Bartoloni (a cura di), Per le strade del mondo. Laiche e religiose tra Otto e Novecento, Bologna, il Mulino, 2007; Ead., Da una guerra all'altra. Le infermiere della Croce Rossa fra il 1911 e il 1945, in Luigi Goglia, Renato Moro, Leopoldo Nuti (a cura di), Guerra e pace nell'Italia del Novecento. Politica estera, cultura politica e correnti dell'opinione pubblica, Bologna, il Mulino, 2006, pp. 149-174; Ead., Donne della Croce Rossa tra guerra e impegno sociale, Venezia, Marsilio, 2005; Ead., Italiane alla guerra. L'assistenza ai feriti 1915-1918, Venezia, Marsilio, 2003; Gennaro Rocco, Costantino Cipolla, Alessandro Stievano (a cura di), La storia del nursing in Italia e nel contesto internazionale, Milano, FrancoAngeli, 2015. D'altra parte la storia dell'assistenza è stata studiata anche da un nutrito numero di professionisti; si vedano Edoardo Manzoni, Storia e filosofia dell'assistenza infermieristica, Milano, Masson, 2000; Cecilia Sironi, L'infermiere in Italia: storia di una professione, Roma, Carocci Faber, 2012; Nicola Ramacciati, Infermieri nello Spedale grande di Perugia. Contesti generali e profili locali dall'Unità d'Italia al fascismo, Perugia, Morlacchi, 2003, anche all'indirizzo web www.researchgate.net/publication/236200798_INFERMIERI_NELLO_SPEDALE_GRANDE_DI_PERUGIA_Contesti_ generali_e_profili_locali_dall'Unita_d'Italia_all'epoca_fascista; Alessandra Fiumi, Infermieri e e 
Nelle pagine che seguono intendo analizzare il processo di costruzione di questa figura lungo il quindicennio seguente la Grande guerra in quanto espressione di una nuova pratica di amministrazione della salute della popolazione. Oggetto dell'indagine saranno soprattutto i discorsi sull'assistente sanitaria — sui suoi compiti, la sua "missione", il suo "modus operandi" — e quelli delle assistenti sanitarie visitatrici. Obiettivo del presente articolo non è solo di inquadrare l'attività di queste donne nel periodo di formazione di questa professione - tra gli anni Venti e i primi anni Trenta - ma soprattutto di indagare l'orizzonte concettuale all'interno del quale esse operavano ${ }^{12}$ e la razionalità che sottostava alla creazione della figura dell'assistente sanitaria visitatrice ${ }^{13}$. Ovviamente questa razionalità non può che essere letta in connessione con l'avvento del fascismo e il suo progetto di "curare la razza". Tuttavia, i discorsi che descrivevano l'assistente sanitaria visitatrice come agente fondamentale di questa opera di "bonifica umana" non erano puramente fascisti né puramente italiani. Anzi, il processo di costruzione di questa figura mostra chiaramente che i paradigmi di gestione delle "risorse biologiche" della nazione, del suo "capitale umano", che sottendevano le politiche sociosanitarie concepite, sostenute e - almeno parzialmente - messe in atto dal regime, pur con tutti i limiti e le discrepanze tra propaganda e realtà ${ }^{14}$, non erano puramente fascisti. Essi avevano le loro radici nell'anteguerra e nell'Italia liberale, ma soprattutto si nutrivano dei discorsi che proliferavano nel particolare humus prodotto dal primo conflitto mondiale e della circolazione transnazionale di idee e pratiche concernenti la "razionalizzazione" dei metodi di gestione della salute della popolazione. Tali paradigmi fanno pienamente parte della genealogia delle politiche sociassistenziali della modernità.

\section{Una genealogia transnazionale}

L'emersione della figura dell'assistente sanitaria visitatrice in Italia va collocata nel quadro delle biopolitiche transnazionali ${ }^{15}$ di governo della salute dise-

ospedale. Storia della professione infermieristica tra '800 e '900, Verona, Nettuno, 1993; Isabella Pascucci, Calogera Tavormina, La professione infermieristica in Italia. Un viaggio tra storia e società dal 1800 a oggi, Milano, McGraw-Hill, 2012.

${ }^{12}$ Joan W. Scott, The Evidence of Experience, "Critical Inquiry”, 1991, n. 4, pp. 773-797.

${ }^{13}$ Illuminanti in questo senso le parole di David Horn che, in un saggio del 1988, proponeva "an alternative approach to the historical study of welfare institutions: an anthropological study of welfare practices in positive terms, which might be able to illuminate their specific rationality. By 'rationality' I do not mean that these interventions were 'rational' as opposed to 'irrational', or that they were part of a general trend of rationalization, but that they obeyed and developed a particular logic": si veda David Horn, Welfare, the Social, and the Individual in Interwar Italy, "Cultural Anthropology", 1988, n. 4, pp. 395-407, qui p. 396.

${ }^{14} \mathrm{Si}$ veda per esempio Domenico Preti, La modernizzazione corporativa: economia, salute pubblica, istituzioni e professioni sanitarie, Milano, FrancoAngeli, 1987.

${ }^{15}$ Alison Bashford, Global Biopolitics and the History of World Health, "History of the Human Sciences", 2006, n. 1, pp. 67-88. 
gnate dalla cooperazione internazionale in campo sociosanitario, che nel primo dopoguerra conobbero un profondo mutamento con l'ingresso sulla scena globale di nuovi organismi internazionali e organizzazioni filantropiche nordamericane. Tra queste ultime l'American Red Cross (Arc) ha raccolto meno interesse da parte degli storici rispetto alla ben più studiata Rockefeller Foundation e al suo International Health Board. Eppure anche questa organizzazione ha avuto un ruolo importante nella strutturazione delle politiche sociosanitarie in Europa a ridosso della Grande guerra e soprattutto nella formazione del personale di salute pubblica, anche attraverso la creazione della Commission for Europe e della League of Red Cross Societies. Portatori dei valori della progressive era, i delegati dell'Arc ritenevano che, grazie al miglioramento del "patrimonio biologico" del vecchio continente per mezzo della trasformazione delle sue istituzioni sociosanitarie, l'Europa sarebbe diventata più stabile economicamente e politicamente, circostanza favorevole per la politica statunitense ${ }^{16}$. È in questo quadro che va collocato l'intervento in Italia, strategico anche in virtù del fatto che il paese era all'origine di un ingente flusso di migranti verso gli Stati Uniti, un "capitale umano" da governare in modo "razionale" 17.

Nella penisola l'Arc era intervenuta subito dopo la sconfitta di Caporetto con l'intento di avviare un'opera di soccorso e di promozione dell'immagine degli Stati Uniti presso gli alleati ${ }^{18}$. Tuttavia, ben presto, ai soccorsi di emergenza ${ }^{19} \mathrm{si}$

${ }^{16}$ Julia F. Irwin, Making the World Safe: the American Red Cross and a Nation's Humanitarian Awakening, Oxford, Oxford U.P., 2013; Ead., Sauvons les Bébés: Child Health and U.S. Humanitarian Aid in the First World War Era, "Bulletin of The History of Medicine", 2012, n. 1, pp. 41-42.

${ }^{17}$ Sebbene di lì a qualche anno il governo nordamericano avrebbe posto limiti severi all'immigrazione proveniente dall'Italia, il Report del lavoro della Commission for Tuberculosis dell'American Red Cross insisteva piuttosto sull'utilità per gli Stati Uniti di migliorare gli standard igienici dei migranti italiani all'origine in modo da rendere questi ultimi più "assimilabili" una volta arrivati a destinazione: American Red Cross, Report of the Commission for Tuberculosis. an Attempt to Establish a Method of International Cooperation in Public Health and Welfare Work. General Report, Roma, Tipografia Bertero, 1919, p. 34.

${ }^{18} \mathrm{Su}$ questa parte del lavoro dell'American Red Cross in Italia si vedano, di Daniela Rossini, Il mito americano nell'Italia della Grande guerra, Roma-Bari, Laterza, 2000, e L'internazionalismo wilsoniano e la propaganda di guerra in Italia, in Ead. (a cura di), La propaganda nella Grande guerra tra nazionalismi e internazionalismi, Milano, Unicopli, 2007, pp. 41-61. Si vedano anche J.F. Irwin, Making the World Safe, cit., e Ead., Nation Building and Rebuilding: the American Red Cross in Italy during the Great War, "The Journal of the Gilded Age and Progressive Era", 2009, n. 3, pp. 407-439.

${ }^{19}$ Per un quadro delle attività dell'American Red Cross relative ai soccorsi di emergenza prestati ai civili e all'esercito si vedano Lavinia Dock e al., History of the American Red Cross Nursing, New York, Macmillan, 1922, p. 858. Sulle attività della Commission for Emergency Relief si veda Paul U. Kellog, Seven Weeks in Italy: the Response of the American Red Cross to the Emergency, Paris, Office of the Commissioner for Europe, 1918; American Red Cross, Summary of Works, Department of Civil Affairs, American Red Cross in Italy, Rome, Department of Public Information, 1918, e Charles Montague Bakewell, The Story of the American Red Cross in Italy, New York, Macmillan, 1920; William Hereford, Report of the Department of Public Information: March 1, 1919, Rome, American Red Cross, 1919. 
era affiancato l'impegno di cooperazione con le autorità italiane e con le organizzazioni private impegnate nel settore della salute pubblica per la costruzione di un sistema di welfare "rispondente a standard internazionali". In questo quadro si colloca la creazione di una Commission for Tuberculosis. Nata dalla proposta del direttore della Commission for Prevention of Tuberculosis in France della Rockefeller Foundation, Livingston Ferrand, e dell'associate director della stessa, William Charles White, invitati in Italia dalla Arc - anche su sollecitazione della Cri - per valutare il da farsi nel campo della tbc ${ }^{20}$, la commissione entrò in funzione nel 1918, sotto la direzione dello stesso White, per promuovere in Italia la messa a punto di strumenti "moderni" di lotta alla malattia sociale, che sarebbero tuttavia tornati utili nel governo di tutti i principali problemi di salute pubblica. Uno dei più accreditati tra questi "strumenti" era proprio l'assistente sanitaria visitatrice. L'educazione igienica era infatti ritenuta un fattore fondamentale di ogni politica di salute pubblica ${ }^{21}$.

Quando gli ufficiali della Tuberculosis Commission dell'American Red Cross, e in particolare la responsabile della sezione nursing, Mary Gardner ${ }^{22}$, incominciarono a prodigarsi per creare un "mercato" per il public health nursing in Italia ${ }^{23}$, rivolgendosi a organizzazioni filantropiche impegnate nella lotta alla tubercolosi e ad autorità locali, la visita domiciliare a scopo assistenziale e ispettivo nelle case del proletariato era una pratica che apparteneva già da tempo alla filantropia, in particolare a quella legata al movimento femminile, le cui principali organizzazioni, l'Unione femminile nazionale e il Consiglio nazionale delle donne italiane (Cndi), erano impegnate nella riforma dell'assisten-

${ }^{20}$ Questa la genealogia della commissione delineata in American Red Cross, "Report of the Department of Medical Affairs", pp. 34 sg., in Hoover Institution Archives (d'ora in poi HIA), American National Red Cross, Commission for Italy, b. 96, cart. 16.

${ }^{21}$ Un corso per la formazione di health visitors che entrassero nelle case per istruire le madri sulle pratiche di allevamento dei bambini era già stato istituito dal Children Bureau della missione dell'Arc. Si veda American Red Cross-Department of Civil Affairs, "American Red Cross, Department of Civil Affairs, January 1, 1918-March 1, 1919. Issued by the Department of Public Information", Roma, American Red Cross, 1919, p. 47, in HIA, American National Red Cross, Commission for Italy, b. 95 cart. 2. Tuttavia, per la formazione di vere e proprie assistenti sanitarie visitatrici si dovette aspettare l'intervento della Commission for Tuberculosis.

${ }^{22}$ Mary Gardner era una vera autorità negli Stati Uniti in materia di public health nursing. Autrice del fortunatissimo Public Health Nursing, New York, Macmillan, 1917, presidente della District Nursing Association di Providence (Rhode Island), Gardner era anche tra le fondatrici della National Organization for Public Health Nurses negli Stati Uniti: si veda Marilyn Bailey Ogilvie, Joy Dorothy Harvey (a cura di), The Biographical Dictionary of Women in Science: Pioneering Lives from Ancient Times to the Mid-20th Century, 2 vol., New York-London, Routledge, 2000, vol. I, p. 485. Sul lavoro di Gardner con gli immigrati di origine italiana si veda Mary E. Thompson, Nurses' Role in Prevention of Infant Mortality in 1884-1925: Health Disparities Then and Now, "Journal of Pediatric Nursing", 2012, n. 5, pp. 471-478. DOI: 10.1016/j. pedn.2011.05.011

${ }^{23}$ Mary Gardner, Supplementary report of the nursing section, in American Red Cross, Report, cit., pp. 47-64, qui p. 56. 
za infermieristica. Anche per questa ragione fu proprio al $\mathrm{Cndi}^{24}$ che la $\mathrm{Com}$ missione si rivolse per aprire la prima Scuola per Assistenti sanitarie visitatrici a Roma, dove ricevette il supporto della marchesa Marianna Denti ${ }^{25}$. Questa scuola fu seguita da quella di Genova, creata con la collaborazione della Lega antitubercolare ${ }^{26}$ e da quella di Firenze — istituita con il supporto dell'Associazione nazionale tra infermiere, nata nel 1919 per difendere gli interessi delle infermiere "diplomate", e del Cndi nella persona di Nerina Gigliucci ${ }^{27}$. A Palermo, invece, una Scuola per Assistenti sanitarie visitatrici venne aperta dal Consorzio provinciale antitubercolare solo nel 1924, nonostante l'Arc avesse tentato a più riprese di crearne una ${ }^{28}$. Infatti l'organizzazione statunitense aveva collocato nella città due delle proprie infermiere sotto il controllo di un comitato italiano supervisionato da Edna Foley, succeduta a Gardner nel maggio del 1919, e aveva inviato due allieve presso la scuola di Roma perché si formassero come assistenti sanitarie visitatrici ${ }^{29}$.

Tutte queste scuole, che non mancarono di incontrare qualche iniziale difficoltà a causa della diffidenza di alcuni medici locali ${ }^{30}$, ricevettero il supporto diretto — finanziario e logistico ${ }^{31}$ — dell'Arc. Come si evince da un rapporto

${ }^{24}$ Sul Cndi si vedano Claudia Gori, Crisalidi. Emancipazioniste liberali in età giolittiana, Milano, FrancoAngeli, 2003; Fiorenza Taricone, L'associazionismo femminile italiano dall'Unità al fascismo, Milano, Unicopli, 1996. Per un quadro del movimento femminile si rimanda ai tanti lavori di Annarita Buttafuoco e in particolare a La filantropia come politica. Esperienze dell'emancipazionismo italiano nel novecento, in Lucia Ferrante, Maura Palazzi, Gianna Pomata (a cura di), Ragnatele di rapporti. Patronage e reti di relazioni nella storia delle donne, Torino, Rosemberg \& Sellier, 1988, pp. 166-187, e a Cronache femminili. Temi e momenti della stampa emancipazionista in Italia dall'Unità al fascismo, Arezzo, Dipartimento di studi storico-sociali e filosofici, Università degli studi di Siena, 1988.

${ }^{25}$ Per un quadro d'insieme sulle prime scuole si veda Atti del convegno per uno scambio d'idee sulle questioni relative all'assistenza agli infermi promosso dal CNDI sezione di Firenze, Firenze, Stabilimenti Grafici A. Vallecchi, 1921.

${ }^{26}$ Gabriella Cantù, A proposito delle Scuole per Infermiere ed Assistenti Sanitarie. La scuola di Genova, "Difesa sociale", aprile 1924, n. 4, pp. 91-93, qui p. 92.

${ }^{27} \mathrm{La}$ Commission for Tuberculosis, attraverso Mary Gardner, era entrata precocemente in contatto con la Sezione Toscana del Cndi, proponendole una collaborazione per l'apertura della scuola di Firenze. Si veda in proposito il manoscritto dell'intervento di Elena French Cini a un incontro organizzato dalla Sezione del Cndi per presentare il lavoro delle assistenti sanitarie, sd., in Archivio Centrale dello Stato, fondo Consiglio nazionale delle donne italiane, b. 6, fasc. 13, s.fasc. 6. La scuola era però stata effettivamente avviata grazie al lavoro di Edna Foley: si veda Edna Foley a Colonel Bartlett, "Report September 12 to October 25", 31 ottobre 1919, p. 1, in HIA, American National Red Cross, Commission for Italy, b. 98, cart. 10.

28 "Report from Miss Gardner", 26 marzo 1919, in HIA, American National Red Cross, Commission for Italy, b. 98, cart. 9.

${ }^{29}$ Edna Foley a Colonel Bartlett, "Report September 12 to October 25", 31 ottobre 1919, p. 1, loc. cit. a nota 27.

${ }^{30}$ Alice Fitzgerald, "Report of nursing activities in Italy", 11 novembre 1919, in HIA, American National Red Cross, Commission for Italy, b. 98, cart. 10.

${ }^{31}$ Al termine della missione statunitense in Italia, su sollecitazione di Edna Foley, la quale aveva insistito perché l'organizzazione lasciasse soldi o personale per completare il lavoro 
di Edna Foley, ne esperirono anche l'influenza culturale: a seguire l'organizzazione dei primi corsi, a tenere le lezioni pratiche e a supervisionare il lavoro sul campo erano infatti nurses dell'American Red Cross ${ }^{32}$. Non solo: gli scambi tra Italia e Stati Uniti (e il mondo anglosassone in generale) in tema di public health nursing non si interruppero con lo smantellamento della missione dell'Arc. Essi continuarono ad alimentarsi attraverso viaggi di studio di infermiere italiane negli Stati Uniti, finanziati tanto dall'Arc, convinta della necessità di dare seguito allo sforzo intrapreso a ridosso della Grande guerra, quanto dalla Rockefeller Foundation $^{33}$. Inoltre, tali scambi proseguirono attraverso la partecipazione di assistenti sanitarie italiane ai programmi di formazione rivolti a public health nurses provenienti da differenti paesi, come quello organizzato a Londra dalla League of Red Cross Societies ${ }^{34}$. Quest'ultimo era nato dalla proposta della responsabile del Nursing Bureau della League, Alice Fitzgerald, proprio con l'intento di creare un nucleo di infermiere formate secondo standard internazionali, che potessero riportare nei rispettivi paesi i metodi e le idee apprese durante il soggiorno a Londra ${ }^{35}$.

avviato, l'Arc aveva donato 36.000 lire per supportare le tre scuole allora in funzione: si veda Ernst P. Bicknell a Livingston Ferrand, Parigi, 26 novembre 1919, in HIA, American National Red Cross, Commission for Italy, b. 94, cart. 14.

${ }^{32}$ Edna Foley a Colonel E.O. Bartlett, 10 novembre 1919, in HIA, American National Red Cross, Commission for Italy, b. 98, cart. 10. L. Dock e al., History of the American Red Cross Nursing, cit., p. 869; Mary Gardner, Servizio delle "infermiere visitatrici". Discorso pronunziato all'inaugurazione delle "Scuole per le infermiere visitatrici" in Roma il 17 marzo 1919 ed in Genova il 2 aprile 1919 da Mary Sewell Gardner Ispettrice generale delle infermiere della Commissione antitubercolare Croce Rossa Americana in Italia, Roma, Tipografia nazionale Bertero, 1919.

${ }^{33} \mathrm{Si}$ veda Italian Noblewomen Visit America, "The American Journal of Nursing", ottobre 1921, vol. 22, n. 1, p. 34. I viaggi di studio erano finanziati da borse offerte dall'Arc, che voleva continuare ad alimentare il rapporto con le infermiere della Cri (si veda Edna Foley a Colonel R.E. Olds, 10 giugno 1920, in HIA, National Red Cross, Commission for Italy, b. 96, cart. 25) e della Rockefeller Foundation (si veda "The Rockefeller Foundation Resume of Nursing, 19131930", 100 Years The Rockefeller Foundation, all'indirizzo web www.rockefeller100.org/items/ show/2212, ultimo accesso 15 settembre 2016. Tra coloro che usufruirono dei viaggi di studio offerti dalla Rockefeller Foundation c'era anche Rosanna Fambri, a capo del Sindacato fascista delle infermiere diplomate.

${ }^{34} \mathrm{Si}$ veda International Courses Offered by the League of Red Cross Societies in Conjunction with Bedford College for Women University of London and the College of Nursing London, Session 1932-3, League of Red Cross Societies, Division of Nursing, Paris, 1933. Si veda Susan McGann, Collaboration and Conflict in International Nursing, 1920-39, "Nursing History Review", (16) 2008, pp. 29-57.

${ }^{35}$ Alice Fitzgerald, "Outline of two possible schemes for the training of public health nurses under the auspices of the League of Red Cross Societies", sd., in HIA, League of Red Cross Societies, b. 1, cart. 16. Dal report del primo anno di questa scuola si apprende che l'allieva italiana che vi era stata formata, Lina Molinari, tornata in Italia aveva avviato uno studio sulla situazione dei dispensari e dell'assistenza in Calabria e si era apprestata a creare dei corsi di igiene domestica e puericultura per ragazze: si veda "Supplement of information circular. News bulletin of activities of graduates of the international Course of Public Health Nursing", p. 4, sd., (circa 1922), in HIA, League of Red Cross Societies, b. 2, cart. 5. 
Negli anni seguenti il numero delle scuole continuò a crescere per lo più sotto l'egida della Croce rossa italiana ${ }^{36}$, affiancata da altre istituzioni o organizzazioni interessate a usufruire dei servizi delle assistenti sanitarie come i Consorzi provinciali antitubercolari - istituiti nel 1919 e resi obbligatori in ogni provincia nel 1927 — la Cassa nazionale per le assicurazioni sociali, l'Opera nazionale maternità e infanzia (nata nel 1925) ${ }^{37}$. La fase della sperimentazione e della libera iniziativa in campo formativo si chiuse nel 1925, proprio nel momento in cui il fascismo consolidava il suo regime, quando il decreto legge 1832 intervenne a ufficializzare e regolamentare le scuole convitto per ospedaliere e quelle per assistenti sanitarie visitatrici, nelle quali si sarebbero dovute ammettere solo professioniste dotate di diploma di infermiera ${ }^{38}$. Questa circo-

${ }^{36}$ Al principio degli anni Trenta la Croce rossa italiana aveva all'attivo scuole per assistenti sanitarie a Roma, Firenze, Milano e Trieste, mentre corsi speciali erano previsti nelle scuole convitto per infermiere gestite dall'associazione a Napoli, Bologna, Milano e Roma. Le scuole di Bari, Catania e Palermo, aperte nel corso degli anni Venti, non superarono invece la fine del decennio: si veda Archivio storico della Croce rossa italiana ospitato presso una delle sedi di Roma, via Bernardino Ramazzini (d'ora in poi ASCRI), b. "Asv, Verbali votazioni 1924-1932, M1 C822/1". Nel complesso il numero delle scuole continuò a crescere nei decenni seguenti. Con gli anni, tuttavia, la Croce rossa italiana avrebbe perso il suo monopolio. Nel 1956 solo 11 delle 20 scuole presenti nella penisola risultavano gestite dall'organizzazione filantropica: C. Sironi, L'infermiere in Italia, cit., p. 161.

${ }^{37} \mathrm{Nel} 1926$ l'Onmi iniziò la sua collaborazione con la Croce rossa per la formazione di assistenti sanitarie di igiene materna e infantile. Per una testimonianza sull'impegno della Cassa nazionale per le assicurazioni sociali nella formazione di queste figure si vedano Attività della Cassa Nazionale per le Assicurazioni Sociali, "Difesa sociale", aprile 1927, n. 4, pp. 100106, qui p. 104; Ettore Levi, Un centro studi e di attività sociale, Roma, Istituto italiano di igiene, previdenza e assistenza sociale, 1925, p. 89. Sull'impegno dei consorzi antitubercolari nella creazione di corsi per assistenti sanitarie si veda Giorgio Cosmacini, Maurizio De Filippis, Patrizia Sanseverini, La peste bianca. Milano e la lotta antitubercolare (1882-1945), Milano, FrancoAngeli, 2004. È probabile che anche l'Ente opere assistenziali fosse impegnato nella formazione di assistenti sanitarie destinate alle colonie per l'infanzia, come testimoniato dalla pubblicazione nel 1934 di un testo a cura del responsabile sanitario dell'Eoa, Gianfranco Capuani, intitolato Nozioni igienico-sanitarie per le assistenti sanitarie di colonia. Corso di lezioni tenute nel maggio giugno 1934 a Novara, Novara, Cattaneo, 1934. Oltre alle scuole gestite dalla Croce rossa e da tutte le organizzazioni elencate, esistevano poi corsi per assistenti sanitarie annessi alle scuole convitto per infermiere che sorgevano all'interno di alcuni ospedali. Si veda per esempio il caso di quella dell'Ospedale di Venezia: Scuola Convitto "Nani" per signorine infermiere, con annesso corso per assistenti sanitarie, Venezia, Scuola tipografica Istituto Manin, 1923. Nel 1948 si contavano 18 scuole per assistenti sanitarie: si veda Elisabetta Vezzosi, L'Onmi a Trieste tra assistenza e social welfare: emergenza post-bellica e tentativi di rifor$m a$, in Anna Maria Vinci (a cura di), Carità pubblica, assistenza sociale e politiche di welfare: il caso di Trieste, Trieste, Edizioni Università di Trieste, 2012, pp. 125-151, qui p. 151.

${ }^{38}$ Il decreto del 1925 venne convertito in legge dal decreto 21 novembre 1929, n. 2330, e in seguito inserito nel testo unico delle leggi sanitarie 27 luglio 1934, n. 1265. Nel 1937, con legge 3 giugno, n. 1084, furono stabilite le norme per l'ammissione alla scuola per assistenti sanitarie. Infine il regio decreto 7 dicembre 1942, n. 1486, approvò il regolamento concernente le norme e i programmi di concorso e promozione per le assistenti sanitarie visitatrici provinciali dipendenti dall'amministrazione della Sanità. Per un quadro d'insieme delle leggi che hanno progressivamente regolamentato la figura dell'assistente sanitaria visitatrice si veda Maria Antonietta Rug- 
stanza contribuì a rendere difficoltoso il reclutamento di allieve per i corsi: gli stessi convitti per ospedaliere, infatti, faticavano a formare un numero sufficiente di professioniste ${ }^{39}$. In attesa di studi su questo tema e di lavori che tratteggino un profilo sociodemografico delle prime assistenti sanitarie visitatrici, questioni che non è possibile qui approfondire, si può ipotizzare che queste scuole faticassero a intercettare le figure per le quale erano state pensate: donne che avessero bisogno di guadagnarsi da vivere e al contempo avessero la possibilità e i mezzi per impegnarsi in un percorso di studi relativamente lungo. Non è forse un caso che molte delle prime diplomate di queste scuole, grazie all'iniziale flessibilità dei criteri di ammissione, provenissero dai ranghi delle infermiere volontarie della Croce rossa, molte delle quali appartenevano alle classi medio-alte ${ }^{40}$. Inoltre, è importante sottolineare che, sebbene quella di assistente sanitaria, come avremo modo di vedere, fosse stata disegnata come una professione attraverso la quale le donne avrebbero potuto esprimere appieno le loro tradizionali "qualità femminili" mettendole al servizio del bene pubblico, essa richiedeva un notevole grado di intraprendenza e di autonomia. Le assistenti sanitarie visitatrici dovevano infatti "andare sole ovunque", come spiegava il resoconto del primo corso per assistenti sanitarie di Palermo, dal quale molte allieve si erano ritirate per paura "delle critiche dell'opinione pubblica" Di conseguenza, dopo un periodo iniziale di slancio, negli anni Venti e Trenta il numero di assistenti sanitarie diplomate e completamente formate crebbe con lentezza, nonostante per loro si andassero aprendo sempre nuove posizioni nei dispensari antitubercolari, negli ambulatori, nelle stazioni antimalariche, nelle colonie, nelle scuole, nell'assistenza di fabbrica, nei consultori e anche nelle nuove organizzazioni assistenziali create dal regime negli anni Venti e Trenta, come l'Onmi, l'Opera nazionale dopolavoro o l'Ente opere assistenziali ${ }^{42}$. Per

geri, L'assistente sanitario, un professionista per la promozione della salute, Spoleto-Perugia, Del Gallo, 2006.

${ }^{39}$ Per esempio le scuole convitto della Croce rossa italiana tra il 1916 e il 1928 licenziarono solo 102 diplomate: si veda "Scuole convitto infermiere Cri. Anno scolastico ottobre 1927-giugno 1928", in ASCRI, b. "Asv, Verbali votazioni 1924-1932 M1 C822/1".

${ }^{40}$ Dallo "Stato di servizio delle A.S. registrato nel libretto personale", redatto a Milano il 23 febbraio 1925 dalla locale sezione assistenti sanitarie della Cri (ASCRI, b. "Asv Milano 19161940 M2 C830/2"), risulta che tra le prime diplomate ci fosse una contessa.

41 "Relazione del primo corso per assistenti sanitarie visitatrici indetto dal consorzio provinciale antitubercolare di Palermo", 1924, in ASCRI,, b. "Asv, Verbali votazioni 1924-1932, M1 C822/1".

${ }^{42}$ Croce rossa italiana, Le infermiere della Croce rossa italiana, in occasione del del $6^{\circ}$ Convegno infermiere, Roma, 25-28 settembre 1928, Roma, snt., 1928, p. 47. Stabilire il numero complessivo di assistenti sanitarie diplomate nei decenni successivi alla guerra non è facile: il numero citato riguarda infatti solo le diplomate delle scuole Cri. Il numero di assistenti sanitarie dovette continuare a crescere negli anni se si considera che nel 1941 l'Opera nazionale maternità e infanzia da sola ne impiegava 463: si veda Domenica La Banca, "La creatura tipica del regime". Storia dell'Opera nazionale per la protezione della maternità e dell'infanzia durante il ventennio fascista (1925-43), tesi di dottorato, Università degli studi di Napoli Federico II, 
esempio, un opuscolo della Cri parlava nel 1928 di "sole" 304 diplomate presso le scuole gestite dall'organizzazione ${ }^{43}$. E tuttavia questa lentezza non era affatto sintomo di scarso interesse per questa figura, tanto più che alla mancanza di assistenti sanitarie diplomate si suppliva spesso con personale volontario. Inoltre, accanto a quella dell'assistente sanitaria nascevano negli anni Venti e Trenta figure professionali o semiprofessionali le quali, ancorché dotate di una minore formazione in campo sanitario, dovevano svolgere compiti simili. Nella galassia del social work italiano nei decenni successivi alla Grande guerra si moltiplicarono, infatti, figure contigue e in parte sovrapponibili a quella dell'assistente sanitaria visitatrice; si trattava di visitatrici volontarie dell'Onmi, maestre vigilatrici, vigilatrici di infanzia, vigilatrici sanitarie, ma anche di segretarie di fabbrica e visitatrici fasciste, le quali, benché più impegnate sul versante dell'assistenza sociale in senso stretto, avevano anche il compito di diffondere l'igiene e la "puericultura razionale"44, e infine - dopo la nascita della Scuola romana - di assistenti sociali. Le idee che erano alla base della creazione della figura dell'assistente sanitaria visitatrice si dimostrarono insomma molto popolari. Nel prossimo paragrafo si proverà a delinearle e a esplorare il contesto nel quale esse emersero.

a.a. 2004-2005, pp. 224, all'indirizzo web www.fedoa.unina.it/811/, ultimo accesso 15 settembre 2016.

${ }_{43}^{43}$ Per chiarire queste affermazioni basterà qualche esempio. Presso la scuola di Torino tra il 1921 e il 1924 si diplomarono 37 allieve, ma già a questa data si registravano le difficoltà di reclutamento che l'avrebbero ben presto condotta alla chiusura: si veda in ASCRI, b. "Asv Torino 1921-1948 N2 C830/11". Le diplomate della scuola di Trieste nel 1937 erano 25, delle quali però solo 10 si erano formate presso una scuola convitto prima di intraprendere il corso: si veda Natalia Rosignoli Jerman, L'assistenza infermieristica a Trieste fra passato e presente, Trieste, Collegio Ipasvi, 1990, pp. 97 sg.

${ }^{44}$ La storiografia ha approfondito soprattutto il ruolo "sociale" e "politico" delle visitatrici fasciste. Storici come Silvia Inaudi e David Horn hanno sottolineato il loro ruolo di intermediarie per l'accesso ai servizi e alle prestazioni socioassistenziali da parte delle famiglie. Altri autori, come Paul Corner o Helga Dittrich-Johansen. hanno sottolineato il loro ruolo politico in quanto agenti di controllo e di indottrinamento. Si vedano a questo proposito Helga Dittrich-Johansen, Le 'militi dell'idea'. Storia delle organizzazioni femminili del partito nazionale fascista, Firenze, Olschki, 2002, pp. 152 sg.; David Horn, Social Bodies: Science, Reproduction, and Italian Modernity, Princeton, NJ, Princeton U.P., 1995, pp. 121 sg.; Silvia Inaudi, A tutti indistintamente. L'Ente Opere Assistenziali nel periodo fascista, Bologna, Clueb, 2008; Paul Corner, Fascismo e controllo sociale, "Italia contemporanea", 2002, n. 228, pp. 381-405. Tuttavia le visitatrici fasciste erano, almeno in teoria, anche incaricate di diffondere l'educazione igienica e l'economia domestica tra la popolazione, come dimostrano i manuali e le conferenze dedicate alla loro formazione. Si vedano per esempio Corso preparatorio per visitatrici fasciste, Novara, Cattaneo, 1940; Wanda Scimone, Nozioni per visitatrici fasciste. Conferenze tenute al Corso per visitatrici fasciste, indetto dal fascio femminile e dalla Croce rossa italiana, raccolte da Wanda Scimone, Padova, Cedam, 1937; Erminia De Benedetti, Economia domestica, fonte di educazione nazionale, Roma, Pallotta, 1935. 


\section{Il governo delle condotte individuali}

"[U]na profilassi sociale ben organizzata, saviamente diretta e vigilata, renderà maggiore il valore materiale e morale del capitale uomo, farà risparmiare enormi capitali e solleverà il prestigio della nazione" ${ }^{\text {"45 }}$. Le parole che il noto malariologo e senatore Ettore Marchiafava scriveva sulla rivista medica "Il Policlinico" nel 1921 condensano bene i discorsi che saturavano il dibattito pubblico sulle politiche sociosanitarie nell'Italia del primo dopoguerra ${ }^{46}$. La "grande carneficina", sostenevano igienisti, riformatori sociali e politici di diverso orientamento, aveva reso drammaticamente evidente che il capitale umano era fondamentale per il perseguimento degli interessi nazionali. Inoltre, la guerra era stata un laboratorio per la sperimentazione di tecniche di governo della popolazione: aumento del controllo su tutti i settori della società, accentramento dell' "unità di comando", gestione centralizzata delle decisioni riguardanti la produzione. Il conflitto aveva offerto un terreno di sperimentazioni dell'intervento attivo dello Stato nella gestione delle risorse biologiche della popolazione che, inedito nel contesto dell'Italia prebellica, non sarebbe venuto meno nel dopoguerra. Come hanno sottolineato gli storici del welfare state in Italia, la Grande guerra, lungi dall'essere un freno, rappresentò infatti un fattore di accelerazione della costruzione di politiche sociosanitarie ${ }^{47}$. Per la prima volta, dopo il conflitto, la Stato si assunse la responsabilità economica dell'erogazione di alcune prestazioni in campo sanitario: esemplare in questo senso il terreno della lotta alla tubercolosi ${ }^{48}$ o quello della previdenza, con la trasformazione della Cassa nazionale di previdenza in Cassa nazionale per le assicurazioni sociali, finanziata anche con contributi statali. Inoltre, nel dopoguerra si moltiplicarono i provvedimenti di assistenza alla maternità e all'infanzia, culminati nella creazione dell'Onmi ${ }^{49}$. Il fascismo, come già si diceva, sviluppò ed espanse queste politiche socioassistenziali, facendone un punto qualificante della propria modalità di gestione del "capitale umano" della nazione, ma non le creò dal nul$1 \mathrm{a}^{50}$. È nel clima postbellico - caratteristico, d'altra parte, non solo dell'Italia

${ }^{45}$ Ettore Marchiafava, La medicina sociale del prof. Ettore Levi, "Il Policlinico. Sezione pratica", 14 marzo 1921, n. 11, pp. 368-370.

${ }^{46}$ Ho esplorato più estesamente questi discorsi in Olivia Fiorilli, La Signorina dell'Igiene. Genere e biopolitica nella costruzione dell' 'infermiera moderna', Pisa, Pisa U.P., 2015.

${ }^{47}$ Lorenzo Gaeta, Antonio Viscomi, L'Italia e lo Stato sociale, in Gerhard A. Ritter, Storia dello Stato sociale, Roma-Bari, Laterza, 1996; Fulvio Conti, Gianni Silei, Breve storia dello Stato sociale, Roma, Carocci, 2011; Gianni Silei, Lo Stato sociale in Italia. Storia e documenti, vol. I, Dall'Unità al fascismo 1861-1943, Manduria, Lacaita, 2003.

${ }^{48}$ Tommaso Detti, Salute, società e stato nell'Italia liberale, Milano, FrancoAngeli, 1993, pp. $151 \mathrm{sg}$.

${ }^{49}$ Michela Minesso (a cura di), Stato e infanzia nell'Italia contemporanea. Origini, sviluppo e fine dell'Onmi 1925-1975, Bologna, il Mulino, 2007.

${ }^{50}$ Maria Sophia Quine, Italy's Social Revolution: Charity and Welfare from Liberalism to Fascism, New York, Palgrave, 2002. 
— che vanno cercate le radici di queste politiche assistenziali. Certamente non tutte le misure proposte ed esaminate nell'immediato dopoguerra vennero effettivamente realizzate. Ciò che è interessante rilevare in questa sede, tuttavia, è che le misure socioassistenziali e sanitarie vagheggiate o effettivamente realizzate nei decenni che seguirono il conflitto erano ispirate da una razionalità comune: l'ottimizzazione delle risorse biologiche della popolazione in vista di una migliore produttività del corpo sociale.

Ottimizzare le risorse biologiche della nazione significava in primo luogo controllare e prevenire le malattie. Tuttavia, l'obiettivo che le politiche sociosanitarie del primo dopoguerra si ponevano era la "piena efficienza" del "capitale umano". La nazione aveva infatti bisogno di "individui fisicamente sani, produttori attivi di ricchezza, procreatori eugenici" ${ }^{51}$. Da tutta la popolazione si doveva ottenere la massima produttività nel lavoro, mentre le donne dovevano anche essere messe in condizioni di "espletare al meglio" la propria "funzione riproduttiva". Insomma, nella nuova temperie del primo dopoguerra, e poi in modo sempre più accentuato dopo l'avvento del fascismo, "la medicina sociale e le discipline in essa coinvolte dovevano abbandonare l'atteggiamento repressivo per passare a quello preventivo"52. Era questa la razionalità delle nuove politiche sociali informate dai dettami di queste discipline. Nel contesto del dopoguerra - quando la parola d'ordine della razionalizzazione risuonava in più ambiti, da quello dell'organizzazione della fabbrica o della casa a quello della pianificazione urbana - l'utopia di una gestione manageriale delle "risorse umane" della nazione, che ne garantisse l'ottimizzazione attraverso un'attenta pianificazione, si manifestava anche nel successo incontrato dall'eugenetica — nella sua versione "latina" 53 — dall'igiene sociale e dall'igiene mentale: discipline fino a quel momento minoritarie, che negli anni successivi al conflitto si istituzionalizzarono, fornendo l'impalcatura teorica alle politiche sociosanitarie via via messe in atto, soprattutto dal regime fascista ${ }^{54}$. Tanto l'eugenetica "latina" - con la sua enfasi sull'intervento "ortogenico" sui corpi attraverso le misure suggerite dalla medicina sociale e la sua diffidenza nei confronti della sterilizzazione - quanto l'igiene sociale o quella mentale, orientate alla pre-

${ }^{51}$ R.B., Sforzo personale e assicurazioni sociali, "Il Policlinico. Sezione pratica", 20 aprile 1925, n. 32, p. 577.

52 Dario Padovan, Bio-politica, razzismo e trattamento degli anormali durante il fascismo, in Francesco Cassata, Massimo Moraglio (a cura di), Manicomio, società e politica, Pisa, Bfs, 2005, pp. 59-82, qui p. 63.

${ }^{53}$ Francesco Cassata, Molti, sani e forti. L'eugenetica in Italia, Torino, Bollati Boringhieri, 2006; Claudia Mantovani, Rigenerare la società. L'eugenetica in Italia dalle origini ottocentesche agli anni Trenta, Soveria Mannelli, Rubbettino, 2004; Claudio Pogliano, L'utopia igienista (1870-1920), in Franco Della Peruta (a cura di), Storia d'Italia. Annali 7. Malattia e medicina, Torino, Einaudi, 1984, pp. 589-633.

${ }^{54} \mathrm{Si}$ veda per esempio Maria Sophia Quine, From Malthus to Mussolini: the Italian Eugenics Movement and Fascist Population Policy, 1890-1938, PhD dissertation, University College London, a.a. 1989-1990. 
venzione e alla restituzione degli "anormali" e dei "deficienti" all'"efficienza"55, si proponevano di agire sul corpo sociale in modo "produttivo" e non repressivo al fine di garantirne uno sviluppo controllato. Queste "discipline" erano accomunate dall'intento di modellare, plasmare gli individui, orientarne i comportamenti e le attitudini in modo da renderli più "sani", "produttivi", "efficienti", al fine di ottenere una miglior "performance" del corpo sociale. Improntare le condotte dell'individuo al "sano vivere" era infatti il miglior modo per controllare e prevenire le malattie e al contempo per ottimizzare la salute pubblica. Per raggiungere questo obiettivo era necessario fare in modo che ciascuno interiorizzasse le norme dell'igiene, summa delle regole del "buon vivere". Se l'educazione igienica non era certo una novità del dopoguerra, in questo periodo essa iniziò a essere individuata come un mezzo imprescindibile per qualsiasi politica di tutela e ottimizzazione della salute pubblica. Inoltre, nel primo dopoguerra, il problema di rendere l'educazione igienica più efficace iniziò a essere affrontato e dibattuto. Alle norme dell'igiene doveva essere data pratica attuazione nella vita quotidiana degli individui, modificando le abitudini più intime e personali: per questa ragione esse non potevano essere imposte $^{56}$. Ciascun individuo doveva piuttosto arrivare a desiderare di divenire un buon amministratore delle proprie risorse biologiche e - nel caso delle donne — di quelle della propria famiglia: solo questo lo avrebbe condotto ad adattare le proprie intime abitudini alle "leggi fondamentali che regolano la vita". Questa nebulosa di idee trovò terreno fertile con l'avvento e l'edificazione del regime fascista, il cui programma di gestione della salute pubblica trovò una prima compiuta sintesi nel "discorso dell'ascensione", pronunciato da Mussolini di fronte alla Camera dei deputati nel 1927. Tale programma aveva al centro l'idea che lo Stato dovesse prendere in carico il problema della salute pubblica con l'obiettivo di "migliorare la razza", non attraverso una "selezione negativa" — come suggerito dall'eugenetica "nordica" — bensì attraverso la "rigenerazione" del corpo sociale, come preconizzato dall'eugenetica "latina". In quest'ottica, i due obiettivi complementari dell'aumento della popolazione e dell'ottimizzazione delle sue "risorse biologiche" dovevano passare per l'incremento delle misure di igiene e assistenza sociale - in special modo quelle rivolte alle donne e all'infanzia - e per l'educazione igienica. La figura dell'assistente sanitaria visitatrice, com'è facile immaginare, trovava agevolmente parte in questo progetto. Sebbene, dunque, questa figura professionale avesse le sue origini fuori dall'Italia e si stesse sviluppando in modo sostanzialmente simile in altri

${ }^{55}$ Francesco Cassata, Il lavoro degli "inutili". Fascismo e igiene mentale, in Id., Massimo Moraglio (a cura di), Manicomo, società e politica. Storia, memoria e cultura della devianza mentale dal Piemonte all'Italia, Pisa, Bfs, 2005, pp. 23-36.

${ }^{56}$ Giuseppe Fidotta, "Per il miglioramento della stirpe". Note sulla propaganda igienico-sanitaria durante il fascismo, "Cinergie", marzo 2013, n. 3, online all'indirizzo web www.cinergie.it/?p=2322, ultimo accesso 7 settembre 2016 . 
paesi europei, essa trovava piena corrispondenza con il progetto biopolitico delineato dal fascismo.

È difficile stabilire se le prime assistenti sanitarie in carne e ossa condividessero in toto questo progetto. Nelle prossime pagine si avrà modo di ascoltare la voci di alcune di loro; appare in ogni caso evidente che molte di coloro che hanno lasciato traccia del loro operato, o che hanno preso pubblicamente parola sulla loro professione, sembravano condividere l'orizzonte concettuale all'interno del quale quest'ultima veniva iscritta.

\section{Il lavoro delle assistenti sanitarie visitatrici}

Le assistenti sanitarie visitatrici lavoravano generalmente alle dipendenze di dispensari antitubercolari, ambulatori, consultori - per esempio quelli dell'Onmi —, stazioni antimalariche, colonie per bambini o preventori, associazioni, leghe e comitati impegnati nella lotta alle malattie sociali che gestivano questo tipo di servizi, scuole e fabbriche. I loro compiti erano diversi, spesso a seconda del contesto di lavoro: il dépistage delle malattie sociali, in particolare la tubercolosi, l'assistenza domiciliare, la mediazione nella distribuzione degli aiuti alle famiglie "bisognose", lo smistamento delle persone nelle istituzioni di cura e assistenza. Tuttavia, compito precipuo di queste professioniste, insieme a tutte queste mansioni, era appunto l'educazione igienica delle persone assistite. Presso le scuole, nelle fabbriche, nei consultori, ma soprattutto nelle case, le assistenti sanitarie dovevano insegnare l'igiene individualmente a uomini, bambini e soprattutto donne: queste ultime, infatti, dovevano essere educate ad assumere il compito di riproduttrici del nucleo famigliare e a diventare massaie "moderne" ed "efficienti", pronte a "ricondurre un relativo benessere nelle famiglie insidiate dal male e dal disordine" ${ }^{57}$ e a trasformare la casa in una fabbrica di lavoratori sani, ordinati, previdenti, stabili e - in definitiva - produttivi.

Sondaggi in differenti archivi hanno mostrato che le fonti sul lavoro svolto dalle assistenti sanitarie visitatrici, soprattutto negli anni di formazione e istituzionalizzazione della professione, che qui ci interessano - ovvero tra la Grande guerra e i primi anni Trenta — sono scarse e frammentate. Tuttavia, il materiale conservato a Roma presso l'Archivio storico della Croce rossa italiana, che come abbiamo visto ha avuto un ruolo da protagonista nella storia di questa figura professionale, offre delle testimonianze interessanti. Presso questo archivio sono infatti custodite alcune relazioni redatte da assistenti sanitarie per descrivere il loro lavoro, che possono rendere un'idea del loro operato in diversi contesti.

\footnotetext{
${ }^{57}$ Irene di Targiani Giunti a Luigi Federzoni, 20 giugno 1924, in ASCRI, b. "Asv, Corrispondenza 1923-1928, N2 C830/14".
} 
Per esempio, un'assistente di fabbrica a Milano, nel descrivere il lavoro svolto tra il 1921 e il 1922, insiste sull'educazione igienica impartita agli operai:

mi preoccupai sempre di sviluppare sia coi consigli, sia alle spiegazioni e in tutti i modi possibili la parte igienico-sanitaria, che era per me la più importante, e che mi pareva anche la più accetta agli operai. Infatti dovetti sempre constatare con mia viva soddisfazione che gli operai prendevano un interessamento speciale alla propaganda igienica e gradivano molto le mie visite a domicilio nei casi di malattia.

La "propaganda igienica" svolta dall'assistente sanitaria aveva in particolare lo scopo di "far conoscere i pericoli dell'alcoolismo e del nicotismo, di far comprendere che cosa è la tubercolosi, come si contrae, e come si può evitare e curare". Inoltre, l'assistente sanitaria si dedicava all'espletamento di pratiche legate "all'invio di ammalati all'ospedale, al ricovero di tubercolosi in sanatorio, all'invio di ragazzi alle cure climatiche o mondane od elioterapiche, all'invio di ragazzi o adulti presso medici specializzati per le visite"58.

Lo smistamento delle persone tra le diverse istituzioni di assistenza e l'ausilio nello svolgimento delle pratiche sono centrali anche nel racconto del lavoro di un'assistente sanitaria rionale, che lavorava in collaborazione con un medico condotto a Bologna principalmente attraverso le visite domiciliari. Nelle 759 visite svolte tra il 1923 e il 1924, specifica la relazione, l'assistente sanitaria racconta di aver praticato terapie e fatto "propaganda igienica", ma anche assolto a compiti che potremmo definire di "assistenza sociale":

Ad esempio fui mandata da una giovane malata di bronco-alveolite, per praticarle delle iniezioni. Trovai una famiglia composta di padre (tubercolotico di guerra) madre alla 18 gravidanza (vizio cardiaco) 6 figli conviventi, 3 dei quali malati. Tutti vivevano in un solo ambiente. Le condizioni economiche disastrose. Mi trovai da principio smarrita. Cominciai con la propaganda igienica e profilattica spiegando i pericoli dell'escreato, le precauzioni indispensabili consigliai un recipiente con calce [...]. Mandai parte dei figlioli al Dispensario, che accordò ad uno dei malati il sussidio delle uova. Il bimbo più piccolo $(6$ anni) non poté essere ammesso alla Colonia dei Cinquanta perché sospetto tubercolotico. Fu così diretto alla clinica pediatrica [...]. Un altro bimbo di 2 anni fu alla Casa del Sole poi alla Colonia di Casaglie ed ora frequenta la scuola all'aperto. La madre, dato alla luce il $18^{\circ}$ figlio, si trova nell'impossibilità di allattarlo, così potei ottenere al Baliatico delle Dame di Carità che la bimba fosse affidata per l'allattamento ad una buona nutrice di campagna. Questi i vantaggi sanitari; in riguardo alle condizioni economiche potei ottenere diversi sussidi ${ }^{59}$.

Come si evince da questo resoconto, l'osservazione delle condizioni di vita, spesso drammaticamente precarie - come le relazioni non mancavano di notare -, e delle pratiche quotidiane delle persone assistite era una parte importante del lavoro delle assistenti sanitarie. Ce ne dà un'idea la "scheda famigliare"

58 "Sommario rendiconto del lavoro svolto nello stabilimento arti grafiche Berardi \& co, in ASCRI, b. "Asv Milano 1916-1940, M2 C830/2".

59 "Relazione dell'assistente sanitaria rionale Costanza Rusconi", in ASCRI, "Asv Bologna 1921-1948, M2 C830/1". 
che le visitatrici rionali del Comitato assistenti sanitarie della Cri di Roma dovevano compilare negli anni Venti. Nella scheda dovevano essere registrate non solo le condizioni di salute dei componenti del nucleo, ma anche le rispettive professioni (con relativi salari e ore di lavoro), le condizioni della casa - comprensive di numero degli ambienti e delle finestre, livello di umidità, servizi - e il modo in cui essa era "tenuta" e abitata. Le domande poste nella scheda erano: quante persone convivono nell'appartamento? Quante dormono nella casa del malato? Quante nello stesso letto ${ }^{60}$. Impedire che gli abitanti delle case che visitavano si radunassero a dormire in pochi letti durante la notte era infatti una delle preoccupazioni più forti delle assistenti sanitarie visitatrici.

Sebbene la visita domiciliare fosse centrale nell'attività di queste professioniste, l'educazione igienica poteva essere da loro realizzata anche mediante corsi e riunioni. Ne danno testimonianza le relazioni sulle attività educative svolte dalle assistenti sanitarie formate nella Scuola di Palermo, creata come si diceva sotto l'egida del Consorzio provinciale antitubercolare, che organizzavano corsi di igiene rivolti a bambini e a madri ${ }^{61}$. I corsi venivano poi seguiti da visite domiciliari che servivano anche a verificare la ricezione degli insegnamenti stessi.

Gli ambiti sui quali si appuntava l'educazione igienica praticata dalle assistenti sanitarie erano i più diversi: "pulizia e igiene della casa, arredamento, riscaldamento, illuminazione, manutenzione, alimentazione"62. I consigli distribuiti da queste professioniste potevano riguardare la "preparazione dei pasti e ordinamento dell'ambiente" 63 , ma anche le tecniche di prevenzione del contagio. Fondamentale terreno di intervento delle assistenti sanitarie visitatrici, in special modo quelle impiegate nel settore dell'assistenza alla maternità e all'infanzia, erano le pratiche di allevamento dei bambini. "Una delle mie maggiori preoccupazioni è stata tra le mamme la diffusione degli inestimabili vantaggi dell'allattamento materno. Ho molto insistito anche sulla necessità di conoscere bene le norme igieniche che regolano la prima infanzia" ${ }^{64}$, spiega per esempio la relazione di un'assistente sanitaria attiva presso un "aiuto materno" di Bologna nel 1924.

Tra le questioni più affrontate dalle assistenti sanitarie visitatrici che hanno redatto le relazioni conservate presso l'archivio della Cri figurano senza dubbio

${ }^{60}$ ASCRI, b. “Asv, Assistenza Onmi 1927-1936, N1 C828”.

${ }^{61} \mathrm{Si}$ vedano "Relazione morale dell'attività della direzione della Scuola Assistenti sanitarie sociali del consorzio provinciale anti-tubercolare di Palermo, novembre 1924-dicembre 1925"; "Relazione morale dell'attività della Scuola Assistenti sanitarie del consorzio provinciale antitubercolare di Palermo, gennaio-dicembre 1926",entrambe in ASCRI, b. "Asv, Verbali votazioni 1924-1932, M1 C82211".

62 "Programma di insegnamento del corso assistenti sanitarie di Croce Rossa Italiana per le materie inerenti la puericultura e alla assistenza alla maternità e all'infanzia, anno scolastico 1929-30", in ASCRI, b. "Asv, Assistenza Onmi 1927-1936, N1 C828".

63 "Clinica pediatrica di Bologna, aiuto materno", 1927, in ASCRI, b. "Asv Bologna 19211968, M2 C830/1”.

64 "Assistente sanitaria dell'aiuto materno. Clinica pediatrica di Bologna", 1924, in ASCRI, b. “Asv Bologna 1921-1968, N2 C830/1". 
quelle attinenti alla nutrizione dei bambini: le raccomandazioni sull'allattamento - preferibilmente al seno - e sulla organizzazione "razionale" delle poppate, che dovevano essere rigorosamente scandite, risultano tra le più frequenti. Centrale nell'educazione igienica impartita dalle assistenti sanitarie era anche la regolazione dei contatti tra adulti e bambini. Frequenti erano per esempio i riferimenti all'importanza di non far dormire i neonati con i genitori. $\mathrm{O}$, ancora, la pulizia dei piccoli - in particolare le tecniche per realizzare correttamente il bagnetto - era un tema frequente di educazione delle madri ${ }^{65}$.

Le assistenti sanitarie visitatrici intervenivano, dunque, sulle abitudini più intime delle persone al fine di correggerle e adattarle alle norme dell'igiene. In questo senso possiamo dire che erano chiamate a svolgere un'opera di dréssage dei comportamenti della popolazione e che l'educazione igienica che veicolavano aveva il carattere di una vera e propria tecnologia disciplinare. Attraverso la regolamentazione e la standardizzazione delle pratiche corporee, l'educazione igienica avrebbe dovuto facilitare quel governo delle condotte individuali che, come abbiamo visto nelle pagine precedenti, era considerato elemento essenziale per l'ottimizzazione delle "risorse biologiche" della nazione. In altri termini, l'educazione praticata dalle assistenti sanitarie si proponeva di disciplinare il corpo individuale in vista della regolazione di quello sociale: in questo senso essa era un esemplare punto di giuntura tra "anatomopolitica del corpo e biopolitica della popolazione"66, per usare una nota formula foucaultiana. Eppure, mentre le norme igieniche che le assistenti sanitarie visitatrici erano chiamate a insegnare avevano un carattere evidentemente disciplinare, queste ultime dovevano diffonderle in maniera flessibile, "insinuandole" in modo efficace nelle vite delle persone assistite.

\section{"Donna soprattutto, che nell'acquistarsi l'affetto e la fiducia dei beneficati è maestra sempre"}

L'educazione profilattica si potrà fare certamente in gran parte a mezzo di conferenze, di corsi d'igiene nella scuola, ma se vogliamo trasformare radicalmente certe abitudini, bisogna indirizzarsi direttamente all'interessato. Non è facile sostituire con le norme igieniche, non sempre agevoli, le abitudini profondamente inveterate. Ed ecco l'opera dell'Assistente Sanitaria, che deve illuminare gli individui, spiegandogli i principi di igiene per adattarli alla sua vita di tutti i giorni, alla casa, alla scuola, al laboratorio. Essa deve avere il buon senso di comprendere la psicologia dell'ambiente dove penetra, il tatto per introdursi e farsi obbedire dalla famiglia, guadagnarsi l'onore di essere l'amica del malato e confidente dei famigliari ${ }^{67}$.

${ }^{65} \mathrm{Su}$ questi temi mi permetto di rimandare a Olivia Fiorilli, Insegnare a vivere e bonificare la società: assistenza infermieristica e discorso igienista nel primo dopoguerra, in Silva Aru, Valeria Deplano (a cura di), Costruire una nazione. Politiche, discorsi, rappresentazioni che hanno fatto l'Italia, Verona, Ombre corte, 2013, pp. 107-124.

${ }^{66}$ Michel Foucault, Bisogna difendere la società, Milano, Feltrinelli, 1998, p. 157.

${ }^{67}$ Carmela Sartori, L'assistente sanitaria nei dispensari antitubercolari. Relazione al convegno nazionale delle assistenti sanitarie, Roma, Federazione italiana per la lotta contro la tubercolosi, 1927, p. 5. 
Le parole che Carmela Sartori rivolgeva alle sue colleghe visitatrici riunitesi in occasione di un congresso tenutosi nel 1927 a latere del secondo Convegno nazionale per la lotta contro la tubercolosi a Milano sono particolarmente utili per inquadrare gli elementi che determinarono il "successo" della figura dell'assistente sanitaria visitatrice. Le norme dell'igiene, come spiegava Sartori, dovevano essere attuate nella vita quotidiana e influire sulle abitudini delle persone, pertanto esse dovevano essere "indirizzate direttamente all'interessato" ed essere adattate alla sua quotidianità: solo in questo modo l'individuo poteva essere messo in condizioni di interiorizzarle. Perché ciò avvenisse era necessario stabilire un contatto diretto, individualizzato, con le persone, acquisirne la fiducia. Le "qualità" che servivano per ottenere questo risultato erano buon senso, intuito, tatto, capacità di entrare in confidenza con le persone. In buona sostanza, quelle tradizionalmente attribuite alla femminilità. Ed era proprio la "femminilità" il "capitale" di cui si supponeva che le assistenti sanitarie fossero naturalmente portatrici. Queste ultime, che dovevano essere donne di classe media, apparivano le migliori agenti di un'educazione igienica calibrata sull'individuo, basata sulla persuasione e non sulla coercizione, proprio perché presumibilmente portatrici delle caratteristiche tradizionalmente attribuite alla femminilità $\mathrm{e}$ in particolare alla femminilità borghese ${ }^{68}$. "Soltanto la donna [...] può ingiungere, con l'esempio, le regole esatte del vivere igienico, che fin d'ora sono state predicate con troppe parole; può correggere, confortare, frenare, incitare; soltanto la donna!"69, spiegava Angiola Moretti, segretaria dei Fasci femminili, durante la conferenza dell'Unione internazionale contro la tubercolosi tenutasi a Roma nel 1928. "Correggere, confortare, frenare, incitare": il tradizionale ruolo di agenti di "redenzione" e "civilizzazione" attribuito alle donne borghesi ${ }^{70}$, tanto più in un paese di cultura cattolica come l'Italia, era dunque messo a lavoro nella costruzione dell'assistente sanitaria come campione di una forma di diffusione dell'igiene basata sulla persuasione. A questo si sommavano le altre "qualità" che facevano parte del repertorio della femminilità borghese, quali la grazia, la dolcezza, la capacità di ispirare fiducia e conforto. Scriveva per esempio Giuseppe D’Ormea, figura di spicco della Lega italiana di

${ }^{68}$ Con il termine "borghese" si fa qui riferimento a un ethos più che a una condizione socioeconomica. Le assistenti sanitarie visitatrici, così come le "infermiere moderne", che i riformatori dell'assistenza raccomandavano fossero donne di "civile condizione", dovevano essere espressione di tale ethos. Su questo punto mi permetto di rimandare a O. Fiorilli, La Signorina dell'Igiene, cit.

${ }^{69}$ Angiola Moretti, Formazione delle infermiere fasciste e loro funzione nelle colonie, negli ambulatori, e nelle altre istituzioni intese come prevenzione antitubercolare, Roma, Tipografia del Littorio, 1928, pp. 8-9.

${ }^{70} \mathrm{Su}$ questo punto la letteratura è sterminata. Mi limito a citare Geneviève Fraisse, Michelle Perrot (a cura di), L'Ottocento, in Georges Duby, Michelle Perrot (a cura di), Storia delle donne in Occidente, Roma-Bari, Laterza, 1996; Lucetta Scaraffia, Anna Bravo, Alessandra Pescarolo, Margherita Pelaja, Storia sociale delle donne nell'Italia contemporanea, Roma-Bari, Laterza, 2001. 
profilassi e igiene mentale e futuro dirigente dell'Onmi, in un pamphlet dedicato al "ruolo della donna nella prevenzione della malattia mentale e nervosa":

La visitatrice dunque - o l'Assistente sanitaria, che dir si voglia — deve in questo campo essere la lunga mano dell'alienista [...]. Quanto più volentieri infatti nelle famiglie [...] sarà accolta la visita e la parola incitatrice di una buona mamma, che sa le vie della dolcezza; o della maestra, che già ebbe nella sua scuola l'infelice di oggi; o magari della levatrice, che nella stessa casa entrò in momenti di ansia e di gioia. [...] Ecco perché è necessario che questo compito di confidente venga assunto, palesemente, non dall'alienista - che pure, ripeto, può e deve ispirarlo e guidarlo - ma dal filantropo, e dalla Donna soprattutto, che nell'acquistarsi l'affetto e la fiducia dei beneficati è maestra sempre. Della Donna che è l'emblema di quell'amore e di quella grazia, che il cristianesimo divinizzò nella figura soave della vergine madre ${ }^{71}$.

Più e meglio dell'alienista, o del medico in generale, figure maschili autoritarie e potenzialmente respingenti, le donne erano ritenute naturalmente in grado di conquistare la fiducia e la confidenza delle persone e quindi suscitarne la "volontaria cooperazione", unico viatico per la piena assimilazione delle norme "igieniche" da parte di queste ultime. Come si può intuire dalle parole che chiudono la citazione di D'Ormea, la cultura cattolica offriva nel contesto italiano, al contrario probabilmente di altri contesti nazionali, un bacino di rappresentazioni della femminilità che potevano essere agevolmente mobilitate nella costruzione dell'assistente sanitaria visitatrice.

Altra "qualità femminile" che poteva essere valorizzata al fine di rendere più efficace l'educazione igienica praticata dalle infermiere era la "praticità". Se il medico era il "detentore" del sapere "teorico" sull'igiene, l'assistente sanitaria doveva esserne l'interprete pratica. Spiegava Irene di Targiani Giunti agli igienisti riuniti in occasione del Congresso della Società italiana di igiene: "questa figura di donna [...] viene col suo patrimonio di concetti pratici e con il suo grande ideale di missionaria"72. Con la sua "praticità femminile" l'assistente sanitaria visitatrice doveva essere in grado di "penetrare" più facilmente nelle case del proletariato, presentandosi non come dispensatrice di ordini o di complesse istruzioni teoriche, bensì come "amica e consigliera", portatrice di un sapere semplice, da trasmettere attraverso l'esempio ${ }^{73}$.

La "penetrazione" nelle case e nell'intimità delle famiglie, che in virtù della costruzione della domesticità come terreno simbolico e materiale della femminilità appariva come missione particolarmente adatta a donne di classe media, era l'asse fondamentale del lavoro delle assistenti sanitarie visitatrici. E non solo perché "sanitarizzare" le abitazioni proletarie era considerato fin dal diciannove-

\footnotetext{
${ }^{71}$ Antonio D'Ormea, L'opera della donna per la prevenzione delle malattie mentali e nervose, Roma, Edizioni Ipas, 1925, pp. 24-25.

${ }^{72}$ Irene di Targiani Giunti, Preparazione delle infermiere per la propaganda igienica: relazione della marchesa Irene di Targiani Giunti, Roma, Sper, 1922, p. 5.

${ }^{73} \mathrm{Si}$ veda per esempio Croce rossa italiana, "Istituzione delle assistenti sanitarie italiane", Sezione di Milano, 1922, in ASCRI, b. "Asv Milano 1926-1940, M2 C830/2".
} 
simo secolo un obiettivo fondamentale delle politiche di "bonifica" del corpo sociale. L'opera di queste professioniste, che, come abbiamo visto, si poteva e doveva dispiegare in vari settori di intervento e luoghi, doveva trovare nell'interno domestico il suo terreno elettivo perché era lì che poteva acquisire "il suo massimo valore, in quanto essa assume[va] carattere più personale" 74 . Dal momento che la casa era costruita simbolicamente come lo spazio del privato - e la stessa educazione igienica contribuiva a (ri)produrre questa idea, costruendo la vita domestica come unico possibile contrappunto al tempo/spazio del lavoro - , essa rappresentava il miglior scenario per un incontro "personale" e "intimo" con le persone. Per questo la casa era considerata il luogo ideale dove praticare una forma di educazione individualizzata e quindi "efficace"75.

Ora, se le caratteristiche attribuite alla femminilità e in particolare alla femminilità borghese, dovevano rendere le donne naturalmente adatte a svolgere un'efficace opera di educazione igienica della popolazione, queste qualità dovevano nondimeno essere raffinate attraverso un'esauriente formazione. Le infermiere visitatrici, come più in generale le "lavoratrici sociali" nei decenni successivi al conflitto, dovevano ricevere un'educazione tecnica che consentisse loro di svolgere appropriatamente la loro "delicata missione" ${ }^{96}$. Cosa le assistenti sanitarie visitatrici dovessero fare per compiere efficacemente tale missione si può dedurre dai manuali redatti per loro, dagli articoli comparsi tra gli anni Venti e i primi anni Trenta su riviste specializzate quali il "Bollettino mensile dell'Associazione nazionale tra infermiere" e "Infermiera italiana" pubblicazione del Sindacato fascista delle infermiere diplomate - , articoli per lo più scritti dalle stesse assistenti sanitarie per le proprie colleghe. $\mathrm{O}$, ancora, è possibile dedurlo dagli stessi resoconti del proprio lavoro redatti da queste professioniste, come quelli menzionati nelle pagine precedenti, nei quali ben si avverte la eco delle direttive che ne organizzavano il lavoro.

Abbiamo visto che le assistenti sanitarie visitatrici dovevano presentarsi come "amiche e consigliere", in modo da guadagnare la fiducia della famiglia. Come scriveva in un tema di fine corso pubblicato su "Infermiera italiana" una diplomanda,

[l'assistente sanitaria] deve essere fornita di buon senso e di tatto: buon senso e tatto nelle indispensabili inchieste domiciliari, cercando di attirar subito la simpatia di chi la riceve, e dando l'impressione di essere di aiuto e consiglio, vedendo subito i bisogni, le tare ereditarie fisiche e morali che affliggono l'assistito, per porvi prontamente rimedio ${ }^{77}$.

${ }^{74}$ B. Guidetti, Ciò che pensa - ciò che si propone - ciò a cui aspira l'infermiera. L'assistente sanitaria in zona di bonifica, "Infermiera italiana", marzo-aprile 1936, pp. 27-28, qui p. 27.

${ }^{75} \mathrm{Si}$ veda per esempio Maria Valenzano, La funzione educativa dell'infermiera sociale, "Difesa sociale", ottobre 1923, n. 10, pp. 157-159.

${ }^{76} \mathrm{Su}$ questo punto si veda D. Horn, Social Bodies, cit.

${ }^{77}$ Nella rubrica Le nostre colleghe di domani, tema di diploma di Laura Agnini, allieva della Scuola Assistenti sanitarie della Cri di Milano, Come l'assistente sanitaria visitatrice, anche se 
Essa doveva inoltre instaurare un rapporto amichevole e confidenziale con le persone assistite, così da farsi ascoltare da queste ultime. Per esempio, l'assistente sanitaria Federica Pittini, già responsabile dei corsi per assistenti sanitarie di Roma e poi di Bologna ${ }^{78}$, e futura autrice di un fortunato Manuale di assistenza sociale, raccomandava dalle pagine di "Infermiera italiana" alle sue colleghe di compilare accuratamente le schede famigliari dopo ogni visita. Infatti:

la madre che nella visita precedente ha parlato con l'Asv amichevolmente, si aspetta che questa si ricordi di lei e dei casi suoi, come si ricorda di un'amica o una buona conoscente. [...] vedendo che l'Asv [con l'ausilio di una scheda ben compilata, nda] ricorda ogni particolare di quanto riguarda il suo bambino, o suo marito, o lei stessa, esclama soddisfatta "La Signorina si ricorda di tutto!" e ne è fiera e si sente importante agli occhi della Asv ed è perciò più disposta ad ascoltarla ${ }^{79}$.

Le parole di Pittini mostrano che alle assistenti sanitarie era ben chiara l'importanza di trovare la "tattica" ${ }^{80}$ migliore per far sì che le persone interiorizzassero le norme igieniche. Non a caso a queste ultime si insegnava a studiare la "psicologia" ${ }^{81}$ degli assistiti. Come spiegava Teresa Biondi al quarto Convegno dell'Associazione italiana per l'igiene nel 1926, la visitatrice

deve studiare l'ambiente, la psicologia dei componenti la famiglia, ma più specialmente quella della madre [...]. Bisogna in primo luogo saper ascoltare, bisogna far parlare questa donna per poter scegliere, volta a volta e caso per caso, il miglior modo per arrivare al cuore ${ }^{82}$.

addetta a servizi specializzati, deve regolare la propria attività, per raggiungere l'indispensabile polivalenza di investigazione e segnalazione, "Infermiera italiana", aprile 1938, n. 4, p. 17.

78 "Corso per infermiere visitatrici di igiene materna ed infantile - sede di Roma, anno 1928-1929", in ASCRI, b. "Asv, Verbali votazioni 1924-1932, M1 C822/1"; si veda anche in ASCRI, b. "Asv Bologna 1921-1948, N2 C830/1".

${ }^{79}$ Federica Pittini, Ciò che pensa - ciò che si propone - ciò a cui aspira l'infermiera. Registrazione delle visite domiciliari, "Infermiera italiana", giugno 1935, n. 6, pp. 18-19.

${ }^{80}$ Erano le stesse assistenti sanitarie visitatrici a usare questa parola. Si veda per esempio la "Relazione morale dell'attività della Scuola Assistenti sanitarie del consorzio provinciale antitubercolare di Palermo, gennaio-dicembre 1926", in ASCRI, b. "Asv, Verbali votazioni 1924-1932, M1 C822/1".

${ }^{81}$ Non a caso spesso nei programmi delle scuole per assistenti sanitarie era previsto lo studio della "psicologia popolare". Si veda per esempio il programma del "Corso assistenti sanitarie 1928-29" di Milano, in ASCRI, b. “Asv, Verbali votazioni 1924-1932, M1 C822/1". I programmi ministeriali per le scuole specializzate per assistenti sanitarie visitatrici pubblicati nel 1938 prevedevano l'insegnamento di nozioni di "psicologia sociale e popolare": Programmi di insegnamento e di esame per le scuole convitto professionali per infermiere e per le scuole specializzate per assistenti sanitarie visitatrici, approvati con decreto ministeriale 30 settembre 1938, Napoli, E. Pietrocola, 1938.

${ }_{82}$ Teresa Biondi (maestra vigilatrice scolastica del Governatorato di Roma), La maestra vigilatrice scolastica e l'importanza della sua opera nella profilassi della tubercolosi, in Associazione italiana per l'igiene (a cura di), Atti del quarto congresso nazionale: Torino 10-19 giugno 1926, Roma, Menaglia, 1926, p. 174-178, qui p. 174. In questo caso, come si intuisce dal titolo, l'autrice non parla delle assistenti sanitarie in senso stretto bensì di una figura ibrida, la maestra 
"Volta a volta e caso per caso": le assistenti sanitarie dovevano dimostrarsi flessibili, adattare se stesse e gli insegnamenti igienici alle circostanze specifiche e alla quotidianità delle persone assistite. A dispetto del fatto che l'educazione igienica poteva ben essere definita, come abbiamo visto, un dispositivo disciplinare, per essere efficaci le assistenti sanitarie che questa educazione dovevano portare "al popolo" dovevano agire sulla dimensione emozionale e su quella del desiderio, dal momento che obiettivo del loro lavoro era far sì che le persone adattassero volontariamente alle norme dell'igiene le proprie condotte più intime. Esse non dovevano presentarsi come figure autoritarie né fare appello alla razionalità delle persone a cui si rivolgevano, quanto piuttosto "parlare al cuore" ${ }^{83}$. Le assistenti sanitarie dovevano insomma conquistare la fiducia delle persone assistite e ingenerare un clima di confidenza che avrebbe consentito loro di trasmettere a queste ultime il "desiderio" di assumere delle condotte di vita sane. A questo scopo erano pertanto invitate a evitare le reprimende e a cercare invece delle leve "positive" 84 .

"[R]enditi conto di ciò che tu devi ottenere, delle persone da cui lo devi ottenere... e poi studia e trova i mezzi necessari per arrivare, ricordati però che non devi scendere se non a rari e temporanei compromessi" ${ }^{85}$ : le parole che Rosanna Fambri, direttrice di "Infermiera italiana" e dirigente del Sindacato fascista delle infermiere diplomate, rivolgeva a un'ideale allieva infermiera, sono una summa del modus operandi dalle assistenti sanitarie visitatrici. Esse non dovevano imporre le norme igieniche attraverso la coercizione o la repressione, ma operare in modo "positivo", approfittando delle circostanze e delle possibilità incontrate ${ }^{86}$. In questo senso possiamo affermare che la razionalità che ne guidava l'opera era pienamente biopolitica. Se con Foucault possiamo definire biopolitico un potere che, avendo a cuore il governo della vita, opera in modo produttivo e non repressivo ${ }^{87}$, allora possiamo dire che il modo in cui le assistenti sanitarie visitatrici dovevano diffondere l'educazione igienica rispondeva a una logica pienamente biopolitica. "Garanzia" di tale modus operandi era il "capitale" primario di queste professioniste, ovvero il loro genere.

vigilatrice appunto, che però ha le stesse competenze dell'assistente sanitaria scolastica a esclusione dell'assistenza ai malati in senso stretto (si vedano pp. $180 \mathrm{sg}$ ).

83 "è al loro cuore che ci si deve rivolgere, non alla loro mente, ancora così piena di pregiudizi attinenti all'igiene, alla medicina, al progresso!", scriveva l'assistente sanitaria B. Guidetti, Ciò che pensa, cit., p. 27.

${ }^{84}$ Elisa Majer Rizzioli, Quasi un romanzo... linee di studio e di pratica per le infermiere, Firenze, Le Monnier, 1926, pp. 13-14.

${ }^{85}$ Rosanna Fambri, La paginetta rosa dell'allieva infermiera, "Infermiera italiana", maggiogiugno 1938, p. 2.

${ }^{86} \mathrm{Si}$ veda Luc Berlivet, Une biopolitique de l'éducation pour la santé. La fabrique des campagnes de prévention, in Didier Fassin, Dominique Memmi (a cura di), Le gouvernement des corps, Paris, Éditions de l'Ehess, 2004, pp. 37-75. Sebbene riferito a un'altra epoca dell'“educazione sanitaria”, il testo offre spunti preziosi su questo punto.

${ }^{87}$ Michel Foucault, La volontà di sapere. Storia della sessualità 1, Milano, Feltrinelli, 2009. 


\section{Conclusioni}

Ho proposto di leggere l'assistente sanitaria visitatrice come una figura paradigmatica delle biopolitiche di governo della popolazione affermatesi negli anni che seguirono alla Grande guerra, non solo in contesto italiano. Il suo lavoro era infatti diretta esemplificazione di uno dei perni di tale politiche: la gestione delle condotte dell'individuo, conditio sine qua non per il miglioramento delle "funzioni vitali" della popolazione nel suo complesso. Come si è avuto modo di vedere, l'assistente sanitaria visitatrice nasceva proprio come risposta alla nuova centralità che il governo delle pratiche quotidiane delle persone stava guadagnando tra le tecnologie di governo della salute del corpo sociale nel primo dopoguerra. Se per ottimizzare le risorse biologiche della popolazione nel suo complesso era necessario coinvolgere il singolo nel "buon governo" della propria salute, l'educazione igienica assumeva infatti un ruolo chiave. L'assistente sanitaria visitatrice emergeva allora come campione di una "nuova strategia" di propaganda igienica calibrata sull'individuo e sulla sua vita quotidiana. La specificità della "missione" dell'assistente sanitaria visitatrice, ovvero quella di "persuadere", "convincere" le persone ad adattare le proprie condotte più intime alle norme dell'igiene, rispondeva perfettamente al paradigma "produttivo" e non repressivo che dava forma alle politiche sociosanitarie nei decenni successivi alla guerra.

In questo quadro il genere, e la sua declinazione in termini di classe, aveva un ruolo fondamentale: come abbiamo avuto modo di vedere, erano proprio le qualità tradizionalmente attribuite alla femminilità — e in particolare alla femminilità borghese - a essere valorizzate nella costruzione discorsiva dell'assistente sanitaria visitatrice. L'analisi di questa figura permette pertanto di gettare luce su uno dei modi in cui il bacino di immagini associate alla femminilità (borghese) è stata messa a lavoro nella costruzione di pratiche e istituzioni del governo biopolitico della popolazione in un periodo in cui sono state costruite le premesse del welfare state in Italia. 